\title{
An Experimental Study of Features Search under Visual Interference in Radar Situation-Interface
}

\author{
Xiao-Li Wu ${ }^{1 *}$, Jing $\mathrm{Li}^{2}$ and Feng Zhou ${ }^{1}$
}

\begin{abstract}
As was found through information interface analysis of target search in fighter radar situation environment, there are many of visual attention problems caused by complex environment and features, which generate visual interference. This paper extracts the interference environment of situation interface and the feature of information matter as the major factors, also and adopts the interference environment, featured items and quantity as three variables to conduct the experimental study on simulation of the feature search fighter information identification. The experimental results showed that the information identification under low and high interference environments revealed to a significant difference. Due to the high interference environment formed by various information presented in the radar situation-interface, only if being forcibly free from interference could the pilots be able to realize the information identification precisely. Three features, i.e., regular shape-single color, irregular shape-single color and irregular shapehybrid color, presented a trend of progressive increase of reaction time, which suggested that irregular shape-hybrid color was the difficult cognition and the long reaction time. The eye movement data also suggested that the target search under high interference environment displayed the longer fixation time, more saccade counts and longer scan path, than under low interference environment. In addition, the first saccade time, the total fixation time and saccade counts of featured items search presented an increasing trend, which is the same as reaction. Therefore, the colors and shapes of featured items exerted a remarkable influence to the judgment of objects target. In conclusion the interference environment and the information matter features both have played the important roles in influence of the information identification in the radar situation-interface. The environment and the features are the design factors, which needed for consideration in the information layout of the complex situation-interface. Thus, a conclusion provides a design guideline to the rational layout and improvement of the complex information interface.
\end{abstract}

Keywords: Radar situation-interface, Goal-seeking, Visual interference, Interference environment, Information matter features, Target identification, Visual displays

\section{Introduction}

In light of the rapid development of industrial design and computer interactive media, visual information interface has become an essential method of information interaction in complex systems. Fighter radar situation environment displayed by digital interactive interface and multi-level data units constitutes a complex dynamic

\footnotetext{
*Correspondence: wuxlhhu@163.com

${ }^{1}$ China College of Mechanical and Electrical Engineering, Hohai University, Changzhou 213022, China

Full list of author information is available at the end of the article
}

system. The digital interactive interface contains a large amount of information that displays and requires pilots to process large-scale information at the same time. That therefore leads to great interference as it is quite difficult for the pilot to cognize during the executive process. The display format information of matter, e.g., shapes and colors, will also lead to some interference to find various purposes. The mentioned above interferences are prone to cause omission, misreading and misjudgment in task execution. Studies have manifested that, in case of emergency, the display format of visual information of pilots 
exerts a remarkable influence on the cognitive performance of information receivers.

\subsection{Literature Review}

\subsubsection{The Radar Situation-Interface}

Battlefield situation is the major resource for pilots to learn about the flight status data, task information as well as threat and security state information. Pilots must grasp distinct, real and complete information of combat situation, so that to have the initiative to the battlefield in hands. Thus, it is clear that the display of information about the battlefield situation is extremely significant. However, multi-level information contents may cause visual interference, which will result on pilots' wrong perception about the information at digital display interface. For example, a pilot may not be able to precisely capture the target due to misunderstanding when guide probability of the acquisition missile capture in radar situation-interface and thus bungle the chance of winning a battle. European and North American National Aeronautics, and Space Administrations have conducted lots of experimental studies on improving the naturalness and effectiveness of man-machine interactions in the pilot cockpit (Mark [1]; Heather et al. [2]). These experiments are aimed to avoid nonconforming to human cognition designs or even wrong designs, to relieve the pilots' burdens and reduce the hazard ratio of flight.

However, the experimental study of target search still needs to be carried in the further. The information display regarding to automatic combat identification system has been studied (Heather et al. [2]). First they applied the testing environment of military simulation and virtual modeling to investigate the hostile and friendly forces as well as the information display formats of visual targets, for example, uniforms and helmets. The investigation results were displayed respectively in pie chart and mesh chart, as well as in separated and integrated information. This paper discussed how these two groups of forms be presented to conduct the identification of combat information as well as the information analysis with combination of information reliability. After obtaining the reliable simulated data, there was found that the display formats of mesh chart and integrated data are more adaptive for combat information identification. Refs. [3-6] have studied the identification performances of the colors, positions and shapes of different symbols and texts, and have obtained a series of valuable results that suit to national conditions. Tong et al. [7] studies controlling machining progress based on information fusion in monitoring computer numerical and has established the structure model from information source. The layout design of fighter radar situation-interface has been evaluated experimentally and has been analyzed through an objective evaluation technology of eye's tracker (Wang et al. $[8,9])$. They also have selected a special scheme of rational layout optimization through the evaluation by eye's moving data indexes. Liu [10] simulated the general operation sequence of enemy attack task in avionics system to conduct interface design on infrared radar system, navigation system, weapon mounting system and flight control system, and evaluated the rationality of interface layout, navigation and graphic symbols by the eye's tracker experiment. Li et al. [11,12] studied the influence of complex digital interfaces on color and shape codes to explore the identification performances under different time pressures. Refs. [13-26] performed physiological research on the information representation and interface design of human-machine interfaces of complex systems.

For all the mentioned above studies, the experimental data are adopted as the main evaluation method to judge the rationality of complex information interface design. Few scholars have set foot in the fields like interference environment and information matter.

\subsubsection{Visual Search Experiment}

Regarding to the experimental study of visual search, the opinion has been held that the occurrence of attention capture basically depends on the significance level of the feature one stimulus relative to other stimuli (Theeuwes et al. $[27,28])$. Then higher the featured significance level of a stimulus that higher the possibility of its generating attention captures. Through experimental observation, Fleetwood $[29,30]$ found that the first factor is the quantity of icons, which influence to the user's visual search, the second is the target boundary, and the last one is the quality and resolution of icons. Patrick [31] has applied the experimental paradigm of visual delay search task to comparatively study of the binding experiment of colors, positions as well as colors and positions. The results showed that the binding experiment had not obviously shortened the search time comparing with the others two groups of experiments. It has been studied that the role of word gap played in visual interference, have adopted the same-different matching task and visual search the time series presented by word gap and stimulus as the variables (Yu et al. [32], and Luo [33]). These two experiments validated that word gap is a necessary and sufficient condition for visual interference derived from context. The researchers have employed the brightness and flash as the way of highlighting to study the symbol's shapes and colors, and testified to the influence of this on search time (Van Orden et al. [34]). The information identifications have been studied in different color codes and spatial positions under multiple information channels (Wickens et al. [35] and Michelle et al. [36]). 
According to the experimental study above, review of visual search for information symbols color, shape, typeface, position as well as icon quantity and quality have possessed a large experimental study basis, and their interactive interference effects have been primarily demonstrated by experiments. Although all of these are basic psychology experiments, they have certain reference value for practical application of radar situation-interface based on which the preliminary presupposition of this experiment could be obtained.

\subsection{Proposed Approach}

Starting from the visual interference of cognitive psychology, this paper conducts an experimental study of fighter situation-interface feature search as next:

Simulate the radar situation-interface of radar air-toground attack display (AD) of F35 fighter (later referred to a radar situation interface);

Extract the associated factors of visual interference;

Apply the technological means of psychological experiment on aspects for example, interference environment and features of information matter;

Carry out the experiment of visual interference on target search;

Analyze the attentional capture and search strategy of identification the different information matters in different interference environments, according to the features of visual selective attention;

Explore the law of the influence interference environment and information matter features on visual search.

\section{Design of Experiment 1}

\subsection{Assumption}

During the process of the information identification interface, visual interferences makes users get into the trouble of target search, which easily causes attention shift. Especially in the case where information is highly concentrated, users can hardly identify, thus generating misperception and attention failure. This experiment conducted multiple-factors of visual search tasks in allusion to the interference environment and information matter features tasks to analyze the influence of different interference degrees and features of visual search. Two assumptions were proposed:

Assumption 1: The more complex the environment of fighter situation-interface than the higher the interference, the longer the time of target search and the higher the error rate

Assumption 2: Visual display format of information matter (regular and irregular shapes, single color and hybrid color) is an important factor to contribute the speed of target identification.

\subsection{Method}

\subsubsection{Materials}

This experiment was planned to simulate the interface of radar situation display of American Fighter F35, and to simplify relevant icon's information of the fighter into experimental conceptual symbols as experimental materials. The interface background is colored in black, and the commonly-used colors of navigation station in green, red, yellow, blue and white. These colors were adopted for the color selection of the item features. The Figure 1 below provides the attack display and the defense display the radar situation for F35 fighter.

The information matters with different meanings in F35 radar defense-situation interface were extracted and drawn by simulation. 12 kinds of information matters were regarded as the featured items of experimental stimulus. Their size was filled with the black box of $6 \mathrm{~mm} \times 7.5 \mathrm{~mm}$, through of combining the shape's features of different information matters, and selecting red and green as the major colors. The features are defined in three types: feature 1 covers the regular-shape (singleshaped, with a vertical line below) single-color presentation individuals; feature 2 covers the irregular-shape single-color presentation individuals; feature 3 covers the irregular-shape (mainly internal deformable based on circle objects) hybrid-color integrated from red and green presentation individuals. See details in Figure 2. The experiment basically discussed the mutual interference degrees with different feature items, i.e., the interference effect of other similar sub features on one sub feature, which was taken as the target.

\subsubsection{Interference Environment}

All these data are all displayed within a circle of radius $78 \mathrm{~mm}$ in the fighter situation-interface. In the circle, there is an attack range limit of $39 \mathrm{~mm}$, which presented both the morphology of the lead plane in the center and the various data of current situation around, which is causing constant interferences to subjects. All of these displays on fighter screen are constant. At the same time, randomly presented in the defensive vision: enemy planes, friendly aircrafts and unidentified objects, are variable. However, we use a simulated defense situationinterface as the fixed vision display instead of the actual situation-interface. This interface displays information what involved in experiment and cannot fix the central visual field.

Consequently, while the stimulus items are presented, space distances are required to be kept around $25 \mathrm{~mm}$ as far as possible. The stimuli object and targets object are 




AD-attack display

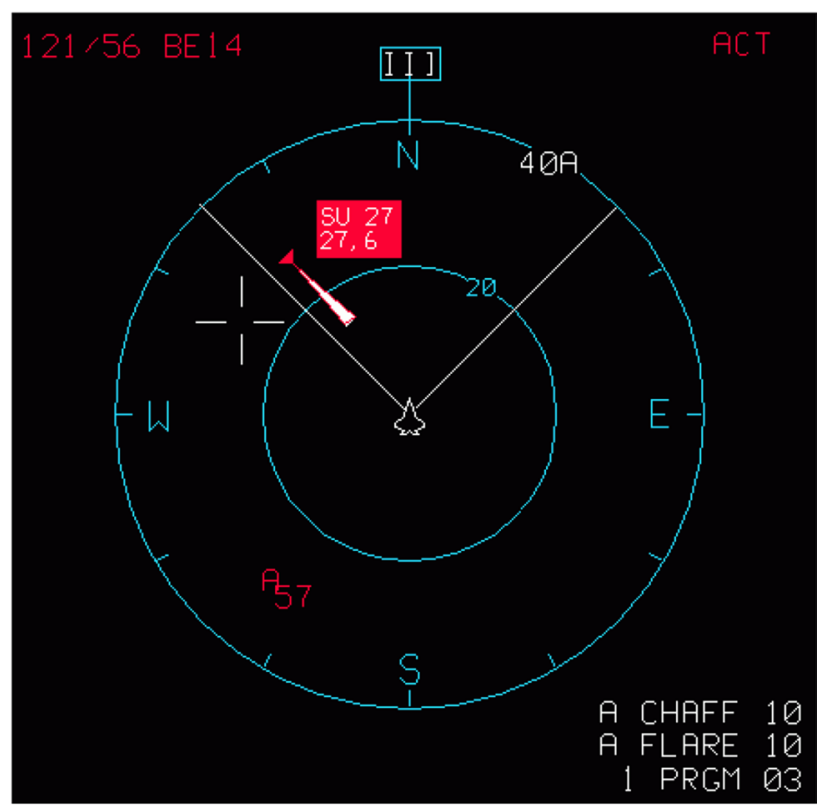

DD-defense display

Figure 1 Radar situation interface of F35 fighter

randomly shown, but all concentrate in the visual range of $6^{\circ}-11^{\circ}$. We have design two experimental display panels, as examination objects both under low-interference and high-interference environments, as indicated in Figure 3.

Based on the analysis above, this experiment adopt the within-group design of $3 \times 2 \times 4$ and apply three factors of stimulus features (1, 2, 3 types) respectively, interference degree (low-interference and high-interference environment), and stimulus item quantity (2, 4, 6, 8 kinds). According to the interference degree, the experiment is divided into two groups, and the interference degrees will appear in the experiment alternatively, each accounts for $50 \%$. Each factor has two tries, each group has $3 \times 4 \times 2$ tries, and 48 tries in total.

\subsection{Experiment Equipment and Procedure}

The experimental procedure was written by the professional psychological experiment development software E-Prime. The experiment was conducted on a computer with a CPU main frequency of $3.0 \mathrm{GHz}$. The stimulus was presented in the center of the 17-inch display with screen resolution $1280 \times 1024$ and luminance $92 \mathrm{~cd} /$ $\mathrm{m}^{2}$. The experiment was conducted in the HumanComputer Interaction Lab of Hohai University. The subject of experiment was presented by 20 undergraduate students in the university: 10 females and 10 males with age between 19 and 23 years old, with normal or corrected vision, and without color blindness or color weakness. The relevant information including gender, age, major and vision was input before the experiment, as indicated in Figure 4.

First, the subjects were required to get familiar with the task environment of the radar situation defense of fighter. In this experiment the situation alternated whole time between the two states as the interference interface: low and high interference. Although some efforts should be made to avoid information interference, in time of conducting the experiment, a first detection item was presented to remember its features (color and shape) to the subjects in a period of $500 \mathrm{~ms}$. After going to the blank screen, various types of stimulus items $(2-10$ in quantity) would be presented to judge whether the feature item previously was observed. If yes that key $\mathrm{V}$ would be pressed, else key $\mathrm{N}$ would be pressed. Also the subjects were prompted that two feature items with the same shape, but different colors are two different feature items. For each person, it took about $15 \mathrm{~min}$ to complete the whole experiment 1.

\section{Design of Experiment 2}

In this eye's movement tracking experiment 2 the featured items, with regular shape-single color, irregular shape-single color and irregular shape-hybrid color were collected, based on the behavior data analysis. The rules 

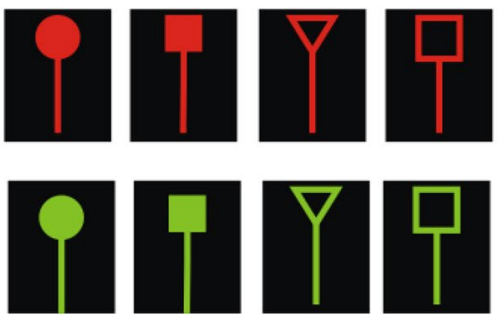

Feature1 (the regular-shape single-color)
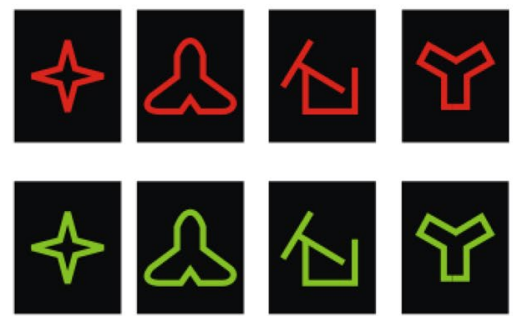

Feature2 (the irregular-shape single-color)
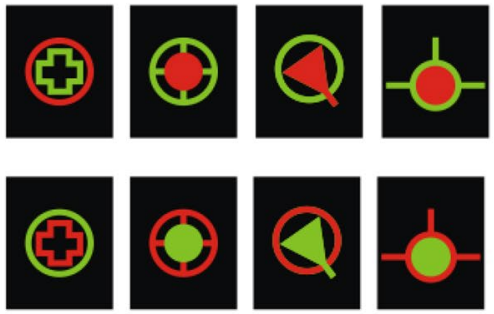

Feature3 (the irregular-shape hybrid-color)

Figure 2 Displays the different feature materials

of subjects' physiological data, including fixation time, gaze plots and fixation points, are discussed later.

The experiment was conducted in the eye's movement tracking laboratory of HHU (Hohai University). The Switzerland-made tobii1X120 eye tracker with a sample frequency of $120 \mathrm{~Hz}$ and gaze location precision of $0.5^{\circ}$ was adopted. The computer with a display pixels of $1280 \times 1024$ (pix), a color quality of 32-bit, and a head movement range of $30 \times 16 \times 20 \mathrm{~cm}$ was accepted. The sight-line gaze location data of the system were delayed to $3 \mathrm{~ms}$ and possessed an ideal gaze and instantaneous display. The system took samples from the eyeballs of subjects every $20 \mathrm{~ms}$ to investigate and collect the data of subjects' eyeball movement.

The experiment was conducted in the Human-Computer Interaction Lab of Hohai University based on collected eye's movement data of 10 participants. This experiment adopts the within-group of design $2 \times 3$ and applies three factors of stimulus features (1, 2, 3 types) respectively. The interference degree (low- and highinterference environment), and 6 stimulus item quantity are not changed. The whole process was conducted according to the first procedure of experiment.

\section{Results}

The experiment 1 mainly analyzed behavioral data of the subjects in the process of target search as next: differences on reaction time under mutual interferences among various factors; error rate under various degrees of interference.

\subsection{Total Reaction Time and Error Rate}

As shown by the results of the $3 \times 2 \times 4$ repeated variance analysis on reaction time, both featured item and quantity had shown main significant effects, and also presence of complex multi-factor interactions. Also as the result of the $3 \times 2 \times 4$ repeated variance analysis on error rate, only the main effect of featured item was significant., and There were significant interactions between feature factor and quantity factor as well as among the three independent variables (as indicated by Table 1).

Under both low-interference and high-interference environments the reaction time and error rates of the subjects were tested when the three different featured items were presented by different quantities. The variance analysis of the reaction time indicated that the main effect of featured items under low-interference environment $(F=24.781, P=0.001, p<0.05)$ and the main effect of feature items under high-interference environment $(F=10.184, P=0.012, p<0.05)$ both had reached the significance level (as indicated by Figures $5(\mathrm{a})$ and (b). The variance analysis on the error rate indicated that the main effect of feature items under low-interference environment $(F=5.297, P=0.047, p<0.05)$ had reached the significance level, while the main effect of feature items under high-interference environment $(F=1.613$, $P=0.275)$ was insignificant, as indicated by Figures 6(a) and (b).

\subsection{Influence of Interference Environment on Different Feature Search}

Posterior multiple comparative tests were conducted by the least significant difference method, including both feature and quantity factor, as indicated by the results shown in Table 2. Significant differences were showed between the presentation of features 3 and 1, and that of feature 2 in reaction time under low and high interference environment. In this way, as showed by the comparisons between the irregular shape-hybrid colored feature item, the regular shape-single colored feature item and irregular shape-single colored feature item, the information matter of relatively complex shape and color usually 


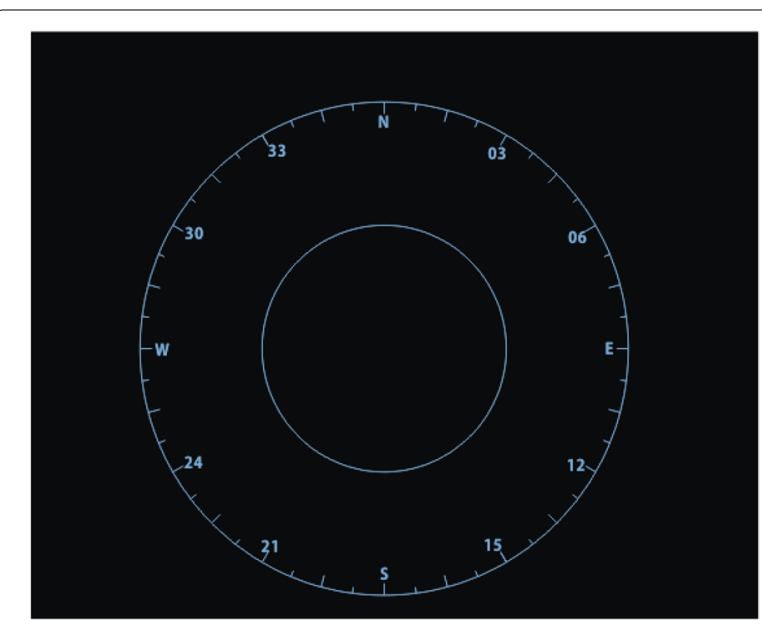

Low-interference environments.

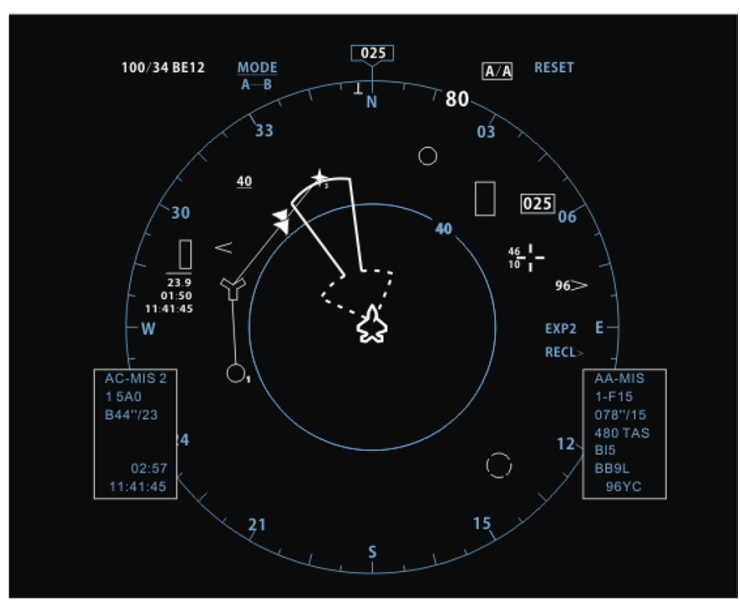

High-interference environments.

Figure 3 Two environments

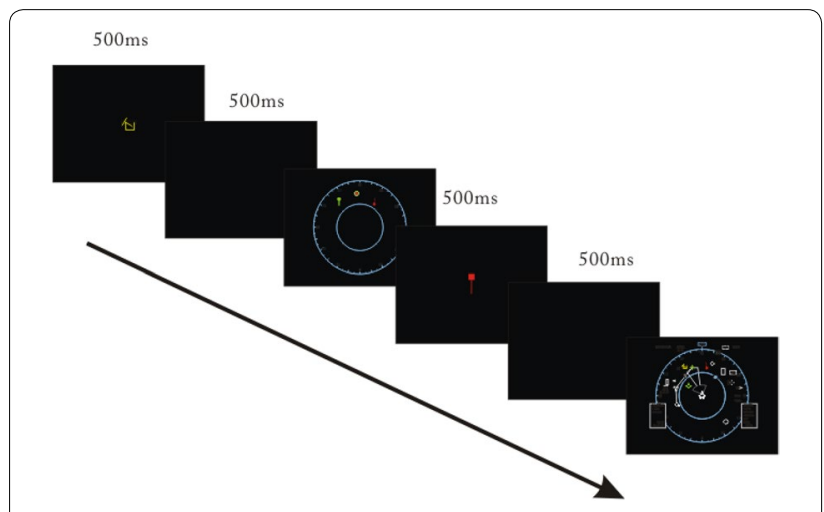

Figure 4 Schematic diagram of the experimental procedure featured of high search difficulty and long reaction time. Thus, in the radar situation environment the error rate under low-interference environment showed significant difference between feature 3 and feature 1. By the time under high-interference environment significant difference was not showed in error rate. This also suggests that under high-interference environment there is certain correlation between the error rate and error factors, which needs to be clarified through in-depth discussions, as indicated by Figures 7(a) and (b).

\section{Discussions}

\subsection{Effect of Featured Items on Visual Search}

Through an experiment of time pressure, the color of features information matter in higher cognitive capacity and speed than of its shape, regarding to studies of information matter features in complex information interfaces (Li et al. [9]). Also the researchers verified the influence of different code schemes on visual search through simulation of the electronic integrated monitoring system of A320. Based on previous studies, the information matter in the radar situation environment was designed in three kinds of different feature items as per the shape and color type features. As shown on Figure 5, data indicated that under low-interference and high-interference environments, with the progressive increase of quantity, feature items showed an obvious trend of progressive increase in reaction and feature 3 (irregular shape-hybrid colored feature item) consumed the longest time. More reaction time needed in high-interference environment than in low-interference environment. Data also indicated that) the error rate presented a law different from that in reaction. In low-interference environment feature items 1,2 and 3 showed a trend of progressive increase, which suggests that regular-shaped single colors are easier to search than irregular-shaped single colors and hybrid colors, and not susceptible to causing misjudgment issues. In high-interference environment the error rate did not show obvious trend of progressive increase, which also suggests that in an environment with multiple interfering object (Figure 6). There are many factors, which may cause errors, so the eye's movement data should be integrated to analyze these interference factors. This may also relate to the randomness of the detection items occurring in the stimulus item, so in the further needed classified statistics of data.

\subsection{Influence of Visual Distraction on Target Search}

In this experiment the high-interference environment basically simulated the complex environment of the fighter situation, and the subjects conducted visual 
Table 1 The main effect on reaction time, error rate, and their interactions for each variable

\begin{tabular}{|c|c|c|c|c|c|c|}
\hline \multirow[t]{2}{*}{ Variation source } & \multicolumn{3}{|c|}{ Reaction time } & \multicolumn{3}{|c|}{ Error rate } \\
\hline & Df & MS & $\mathbf{F}$ & Df & MS & $\mathbf{F}$ \\
\hline Interference & 1 & 21530.157 & 0.129 & 1 & 0.000024 & 0.003 \\
\hline Feature & 2 & 967379.164 & $11.634^{* * *}$ & 2 & 0.22 & $3.956^{*}$ \\
\hline Quantity & 3 & 443387.417 & $3.772^{*}$ & 3 & 0.013 & 2.060 \\
\hline Interference $\times$ Feature & 3 & 652096.162 & $7.563^{* *}$ & 3 & 0.015 & 2.514 \\
\hline Interference $\times$ Quantity & 4 & 337923.102 & 2.756 & 4 & 0.010 & 1.469 \\
\hline Feature $\times$ Quantity & 5 & 652984.116 & $28.251^{* * *}$ & 5 & 0.017 & $3.743^{*}$ \\
\hline Interference $\times$ Feature $\times$ Quantity & 6 & 547741.789 & $23.603^{* * *}$ & 6 & 0.014 & $2.948^{*}$ \\
\hline
\end{tabular}

Note: ${ }^{*} p<0.05,{ }^{* *} p<0.01,{ }^{* * *} p<0.001$



a Under low-interference environment

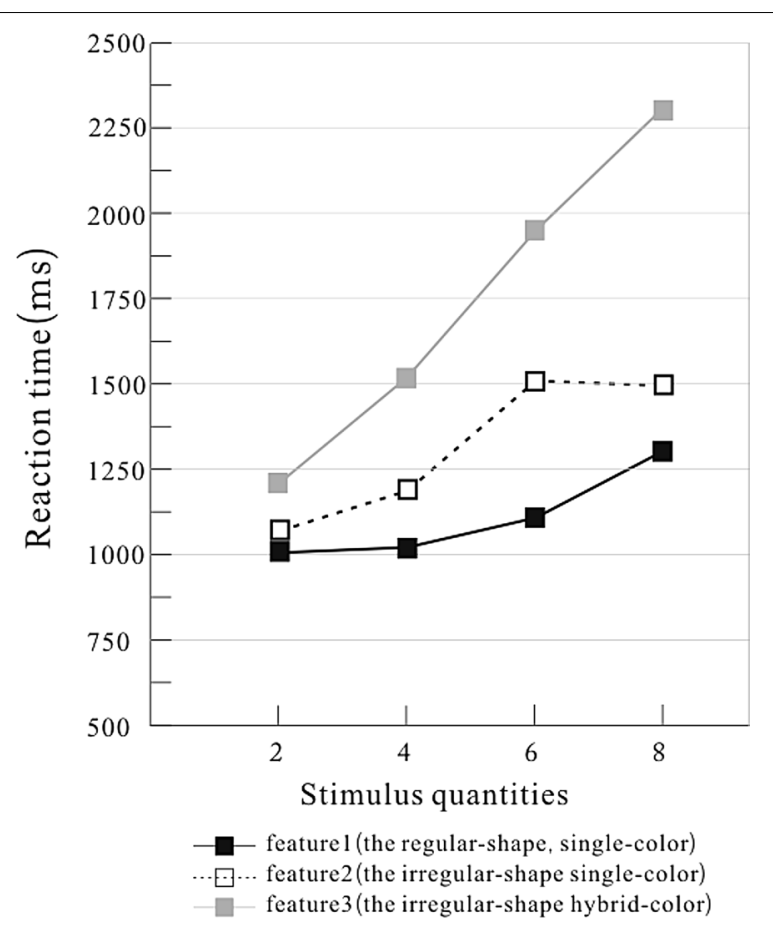

b Under high-interference environment

Figure 5 Reaction time of the three feature items under different interference environment

search in an interference-free environment. Previous experimental studies on interference basically set interference items through sub-feature. In this experiment the single interface (low interference) and real simulation scene (high interference) were employed as variables, and the data showed the significant influence in reaction (Figure $7(\mathrm{a})$ ). This suggests that the complexity of radar situation environment significantly interferes with the identification of information matter, which needs in-depth studies. As indicated by the error rate data (Figure 7(b)) there was no regular change. Also it suggests that error factors are relatively complex and the general law cannot be derived only from interference and feature. However, multi-angle analysis is needed.

\subsection{Influence the Existence of Detection Items on Target Search}

During target search by the subjects on the stimulus feature, the detection items will appear or disappear randomly accounting for $50 \%$ respectively. Statistics show that the reaction time was taken when the target object 


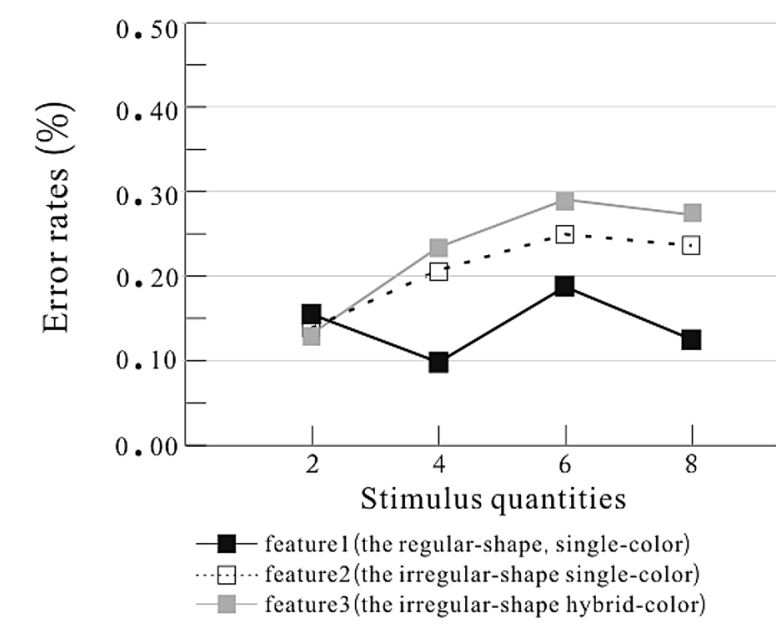

a Under low-interference environment

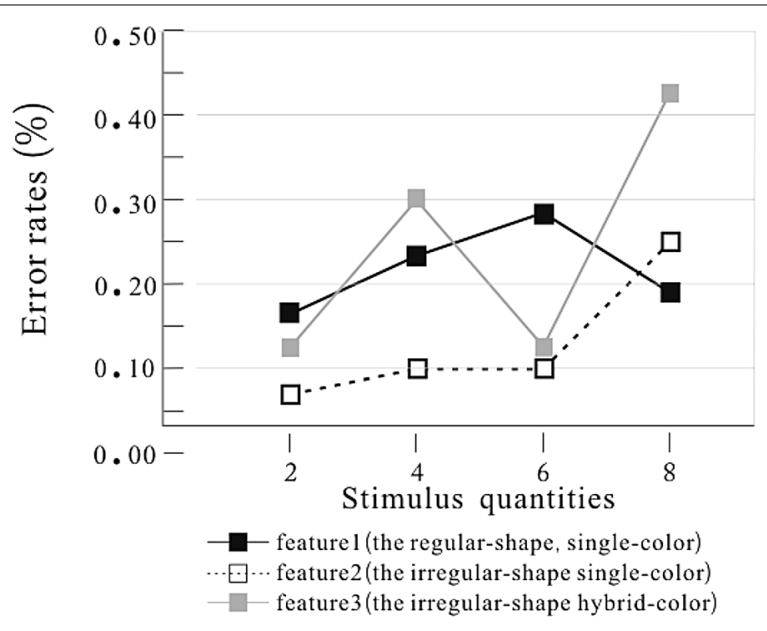

b Under high-interference environment

Figure 6 Error rates of the three feature items under different interference environment

Table 2 Posterior multiple comparative test by the least significant difference method

\begin{tabular}{|c|c|c|c|c|c|c|c|c|}
\hline & \multicolumn{2}{|c|}{ Evaluation index } & \multicolumn{3}{|l|}{ Low interference } & \multicolumn{3}{|l|}{ High interference } \\
\hline & Feature (I) & Feature $(J)$ & Mean error (I-J) & Standard error & $\mathbf{P}$ & Mean error (I-J) & Standard error & $\mathbf{P}$ \\
\hline \multirow[t]{6}{*}{ Reaction time } & Feature 1 & Feature 2 & -207.65600 & 106.810958 & 0.100 & -212.63625 & 141.720018 & 0.184 \\
\hline & & Feature 3 & $-729.72050^{*}$ & 106.810958 & 0.000 & $-628.72200^{*}$ & 141.720018 & 0.004 \\
\hline & Feature 2 & Feature 1 & 207.65600 & 106.810958 & 0.100 & 212.63625 & 141.720018 & 0.184 \\
\hline & & Feature 3 & $-522.06450^{*}$ & 106.810958 & 0.003 & $-416.08575^{*}$ & 141.720018 & 0.026 \\
\hline & Feature 3 & Feature 1 & $729.72050^{*}$ & 106.810958 & 0.000 & $628.72200^{*}$ & 141.720018 & 0.004 \\
\hline & & Feature 2 & $522.06450^{*}$ & 106.810958 & 0.003 & $416.08575^{*}$ & 141.720018 & 0.026 \\
\hline \multirow[t]{6}{*}{ Error rate } & Feature 1 & Feature 2 & -0.06450 & 0.029148 & 0.069 & -0.08025 & 0.064618 & 0.261 \\
\hline & & Feature 3 & $-0.09250^{*}$ & 0.029148 & 0.019 & -0.11275 & 0.064618 & 0.132 \\
\hline & Feature 2 & Feature 1 & 0.06450 & 0.029148 & 0.069 & 0.08025 & 0.064618 & 0.261 \\
\hline & & Feature 3 & -0.02800 & 0.029148 & 0.374 & -0.03250 & 0.064618 & 0.633 \\
\hline & Feature 3 & Feature 1 & $0.09250^{*}$ & 0.029148 & 0.019 & 0.11275 & 0.064618 & 0.132 \\
\hline & & Feature 2 & 0.02800 & 0.029148 & 0.374 & 0.03250 & 0.064618 & 0.633 \\
\hline
\end{tabular}

Note: * represents the reaching level of significance (mean error $>0.05$ )

has been searched out. It is far less than that of when the target object has not been searched out i.e., when there are no detection items, more time will be taken by the subjects on target search. Statistics on various interference environments indicate that when there are no detection items, or when target search takes a longer time, the reaction time shows a trend of progressive increase with the differences of information matter features. When there are detection items it's easy to find the target, and there is no obvious law in reaction, as indicated by the results provided in Table 3.

\subsection{Comparison of Heat Maps and Gaze Plots under Different Interference Conditions}

Figure 8 extracts the heat maps and gaze plots with the same featured item (irregular-shape single color) from eye movement experiment 2 , under low and high interference environments, respectively. Influenced by different degrees of interferences, the overplayed gaze plots of 10 subjects were obviously different. The average saccade counts 4.1 under low interference environment and 8.2 under high interference environment. Therefore, different 


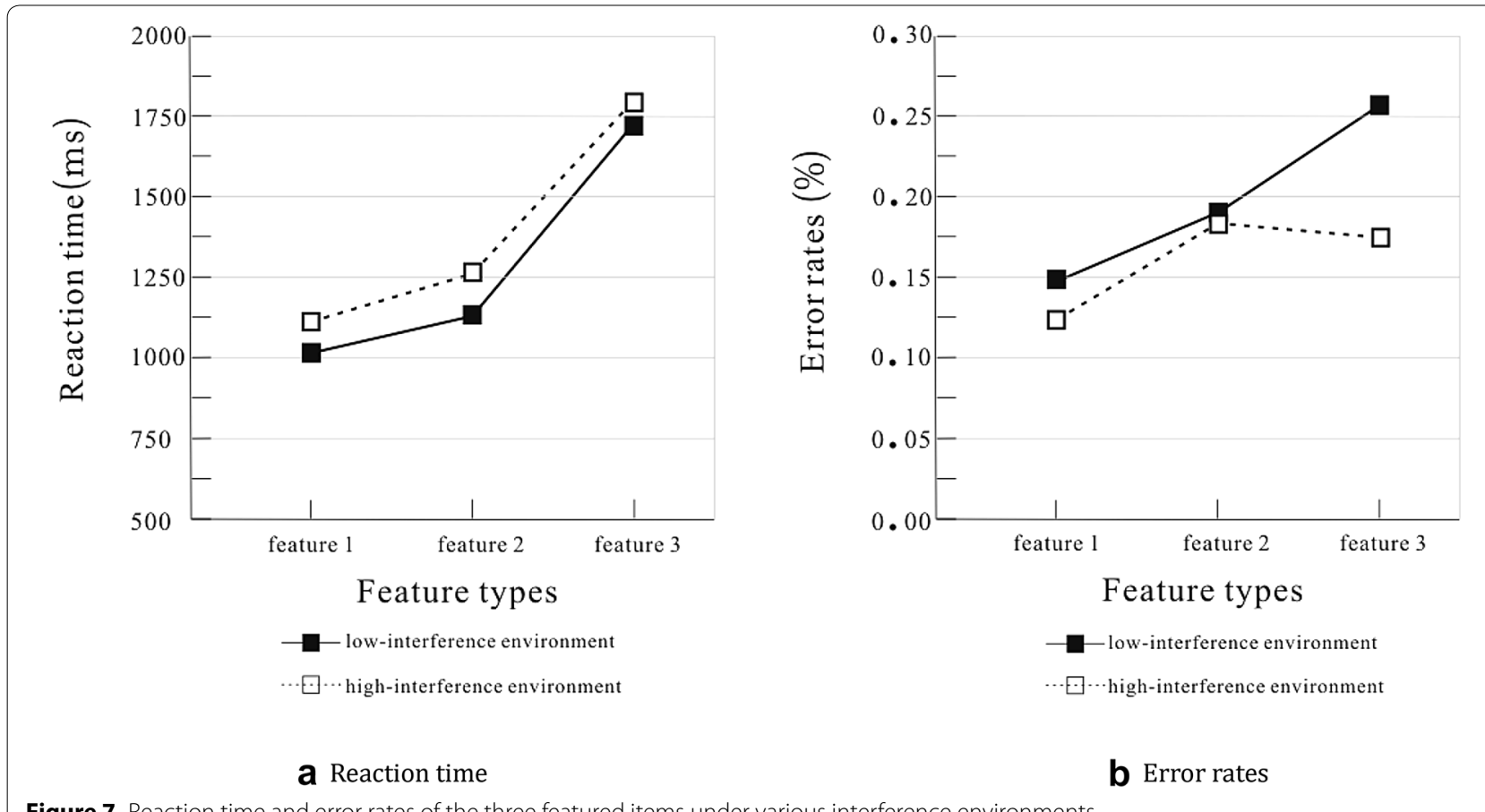

Figure 7 Reaction time and error rates of the three featured items under various interference environments

Table 3 Influence of the existence of detection items on target search

\begin{tabular}{|c|c|c|c|c|c|c|c|c|c|}
\hline \multirow[t]{3}{*}{ Variable } & & \multicolumn{4}{|c|}{ Reaction time } & \multicolumn{4}{|c|}{ Error rate } \\
\hline & & \multicolumn{2}{|c|}{ With detection items } & \multicolumn{2}{|c|}{ Without detection items } & \multicolumn{2}{|c|}{ With detection items } & \multicolumn{2}{|c|}{$\begin{array}{l}\text { Without detection } \\
\text { items }\end{array}$} \\
\hline & & $M$ & SD & $M$ & SD & $M$ & SD & $M$ & SD \\
\hline \multirow[t]{4}{*}{ Low interference } & 2 & 1203.833 & 452.636 & 1164.158 & 393.707 & 0.428 & 0.222 & 0.053 & 0.229 \\
\hline & 4 & 1460.474 & 862.011 & 1499.526 & 476.553 & 0.496 & 0.368 & 0.105 & 0.315 \\
\hline & 6 & 1656.895 & 707.531 & 1930.529 & 816.107 & 0.478 & 0.316 & 0.235 & 0.437 \\
\hline & 8 & 2072.950 & 1306.35 & 2501.100 & 1348.64 & 0.444 & 0.250 & 0.300 & 0.47 \\
\hline \multirow[t]{4}{*}{ High interference } & 2 & 1048.200 & 342.774 & 1375.100 & 1029.71 & 0.366 & 0.150 & 0.100 & 0.308 \\
\hline & 4 & 1498.350 & 666.334 & 1587.684 & 734.76 & 0.366 & 0.150 & 0.421 & 0.496 \\
\hline & 6 & 1845.550 & 595.438 & 2034.900 & 774.488 & 0.41 & 0.200 & 0.050 & 0.224 \\
\hline & 8 & 2520.400 & 1469.76 & 2551.250 & 2908.8 & 0.47 & 0.300 & 0.600 & 0.503 \\
\hline
\end{tabular}

interferences under high interference environment had influenced significantly by the reaction time on subjects to search the target. In addition, the heat map under low interference environment revealed to the focused attention of subjects, but a distraction under high interference environment. Moreover, the attentions of some subjects were even transferred to the data information display in left bottom. The host icon in the middle and mileage display in the right, indicated the attentions, which were distracted during quick search for targets.

\subsection{Influence of Different Featured Items on Fixation Time}

We can analysis the feature areas which are more noticed by participants from point of eye gaze. Creating several AOIs (area of interest) is straightforward and covered in the user's manual so this post will focus on features that are less commonly used. We'll also present a few tips and tricks for designing and analyzing with AOIs.

We decide on the proper balance between selectivity and sensitivity which the gaze data around the feature items to include in the AOI calculation. Smaller AOIs increase selectivity and exclude more extraneous gaze at 


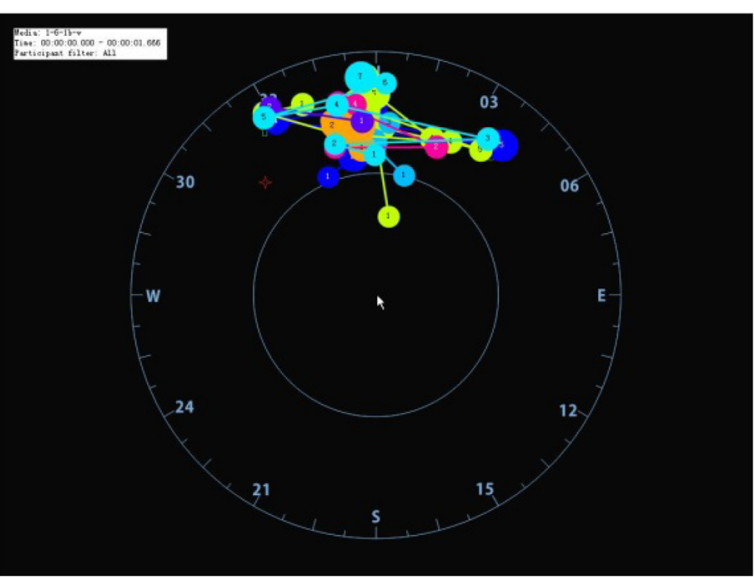

a Gaze plot of low-interference

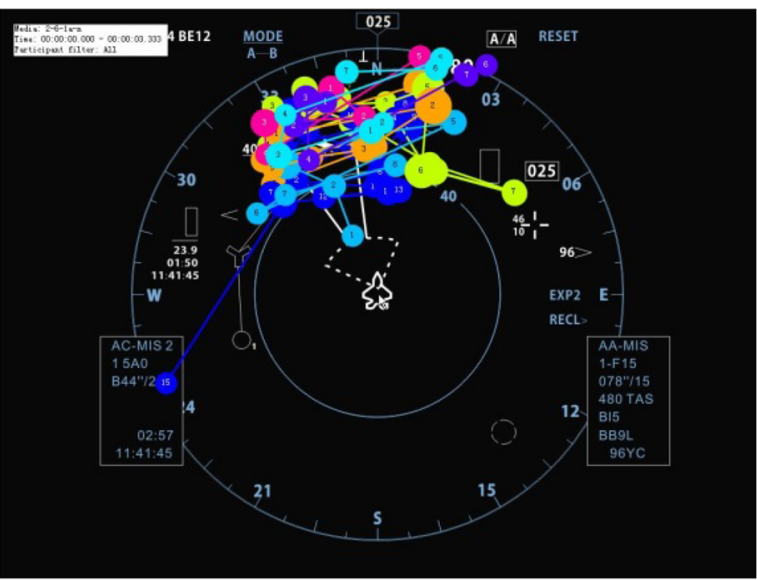

b Gaze plot of high-interference

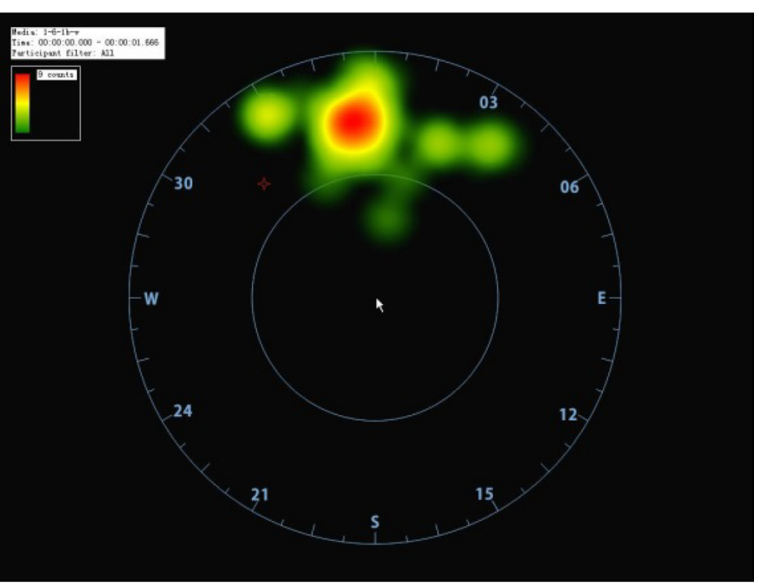

C Heat map of low-interference

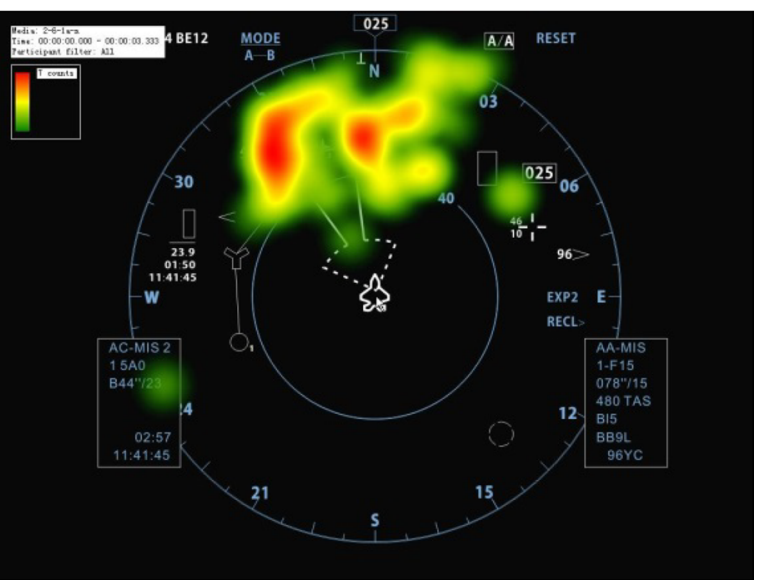

d Heat map of high-interference

Figure 8 Gaze plot and heat map in different environment

the risk of losing some actual valid looking. Larger AOIs increase sensitivity and may include more extraneous data while potentially capturing more of the actual looking to target object and interferent. Therefore, in the featured items as the analytic objects collects the gaze plots contained three different featured items under high interference and divided four AOIs (area of interest) based on the area containing target object and interferent, as it is shown in Figure 9. On this AOI 1 is the location of the featured item. The eye movement data recorded the first fixation time, fixation duration and fixation points.

The data in Table 4 suggested that featured item 1, 2 and 3 presented an increasing trend, with the trend of reaction time showed in the behavior data. Specifically, the fixation time of feature item 1 was the shortest, and the fixation points were aggregated in AOI 1; the fixation

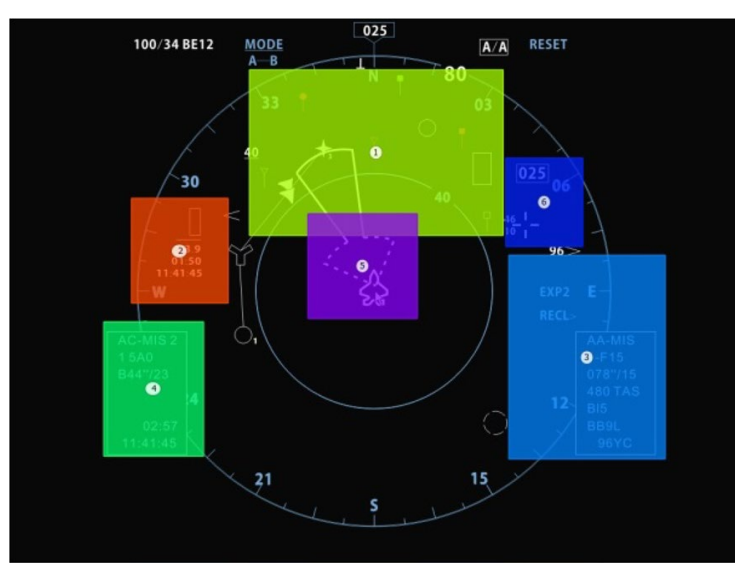

Figure 9 Division of $\mathrm{AOl}$ area 
Table 4 Fixation time in $\mathrm{ms}$ and fixation points with otherwise featured items in different AOI areas

\begin{tabular}{|c|c|c|c|c|c|c|c|c|c|}
\hline \multirow[t]{2}{*}{ AOI } & & \multicolumn{2}{|c|}{ AOI 1 (target) } & \multicolumn{2}{|l|}{ AOI 2} & \multicolumn{2}{|l|}{ AOI 3} & \multicolumn{2}{|l|}{ AOI 4} \\
\hline & & Mean & Sum & Mean & Sum & Mean & Sum & Mean & Sum \\
\hline \multirow[t]{3}{*}{ Time to first fixation } & Feature 1 & 2 & 21 & - & - & - & - & - & - \\
\hline & Feature 2 & 30 & 300 & - & - & - & - & 1061 & 3184 \\
\hline & Feature 3 & 69 & 693 & 805 & 805 & 1227 & 3680 & - & - \\
\hline \multirow[t]{3}{*}{ Total fixation duration } & Feature 1 & 685 & 6846 & - & - & - & - & - & - \\
\hline & Feature 2 & 1303 & 13032 & - & - & - & - & 128 & 383 \\
\hline & Feature 3 & 1308 & 13081 & 167 & 167 & 257 & 770 & - & - \\
\hline \multirow[t]{3}{*}{ Fixation count } & Feature 1 & 3.2 & 32 & - & - & - & - & - & - \\
\hline & Feature 2 & 6.2 & 62 & - & - & - & - & 1 & 3 \\
\hline & Feature 3 & 6.2 & 62 & 1 & 1 & 1.67 & 5 & - & - \\
\hline
\end{tabular}

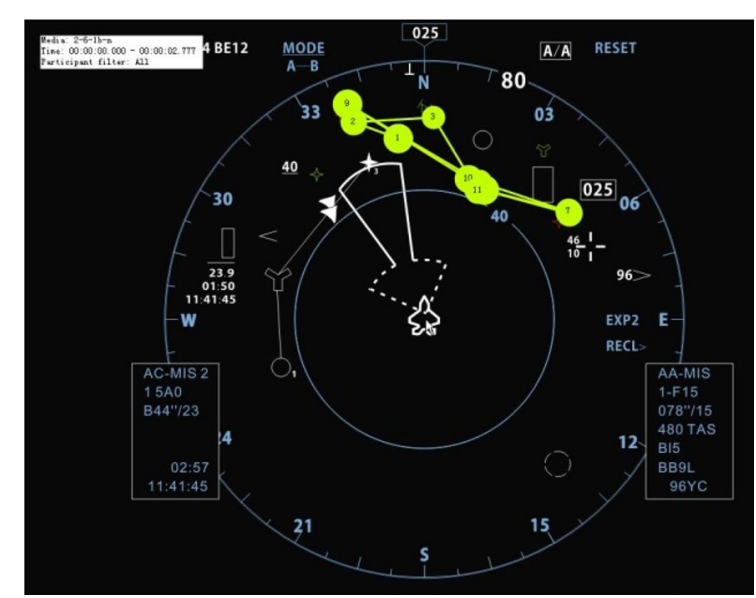

Figure 10 Path of featured item search by a subject (The irregular-shape single-color)

points of featured item 2 and 3 dispersed in AOI 2, AOI 3 and AOI 4, which explained that the interference features in these areas influenced the search tasks of irregularshape single color and irregular-shape hybrid color. Also, as indicated the search of task, regular-shape single color was fast and freed from environmental interference.

\subsection{Analysis of Error Causes}

According to the distribution of error rates in Figure 6, the relationship between error factors and interferences was further analyzed by combining the eye movement data. Featured item 2 and 3 with high error rates were selected as the analytic objects. Figures 10 and 11 shows the gaze plots of two subjects, respectively. Table 5 is the simulation graph, without probe items of the featured items (irregular-shape single color); the target object of featured items was A-4, and the featured items presented by simulation materials were A-1, A-2, A-3, A-4, A-5, A-6, A-7, A-8. Figure 10 shows that the subject displayed

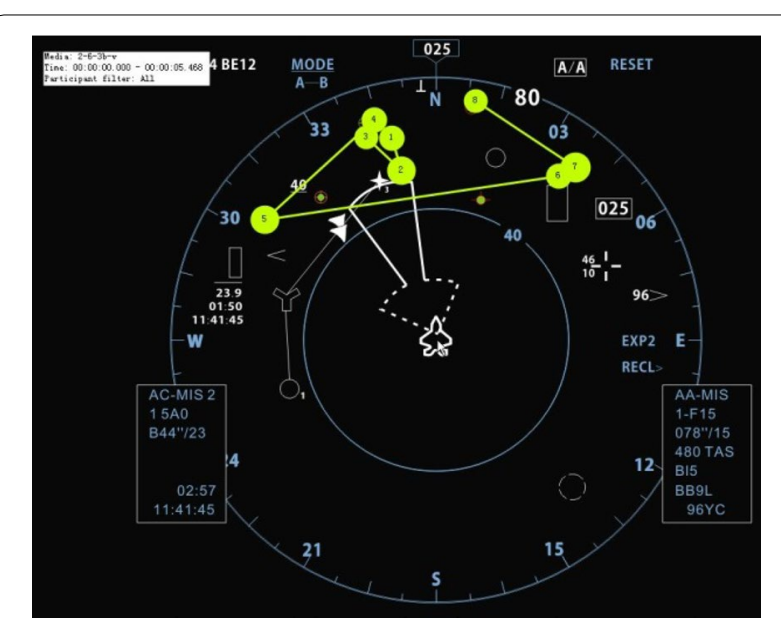

Figure 11 Path of featured item search by a subject (The irregular-shape hybrid-color)

repeated saccades toward three red non-target objects, with total saccade counts of 11 and a reaction duration lasting of $1366 \mathrm{~ms}$ (The reaction time $=1517.806 \mathrm{~ms}$, The error rates $=0.278$ ). It indicated that the subject was not influenced by the other non-target objects, and different interference information in interference environments. Probably, the error was caused by the neglecting of the features of original target objects when distinguishing the features with the same color. It further explained the interference of the same color, which was more likely the result of errors. Figure 11 showed the simulation graph, with probe items of featured items (irregular-shape hybrid color). The featured item of target object was B-1, and the feature items presented by simulation materials were B-1, B-2, B-3, B-4, B-5, B-6, B-7, B-8. Figure 11 shows that it is going through 8 saccades. The subject was not affected by the interference environment and quickly restored to search for the similar featured items. The subject displayed a longer fixation time and finally remained 
Table 5 Target object and the featured items presented by simulation materials

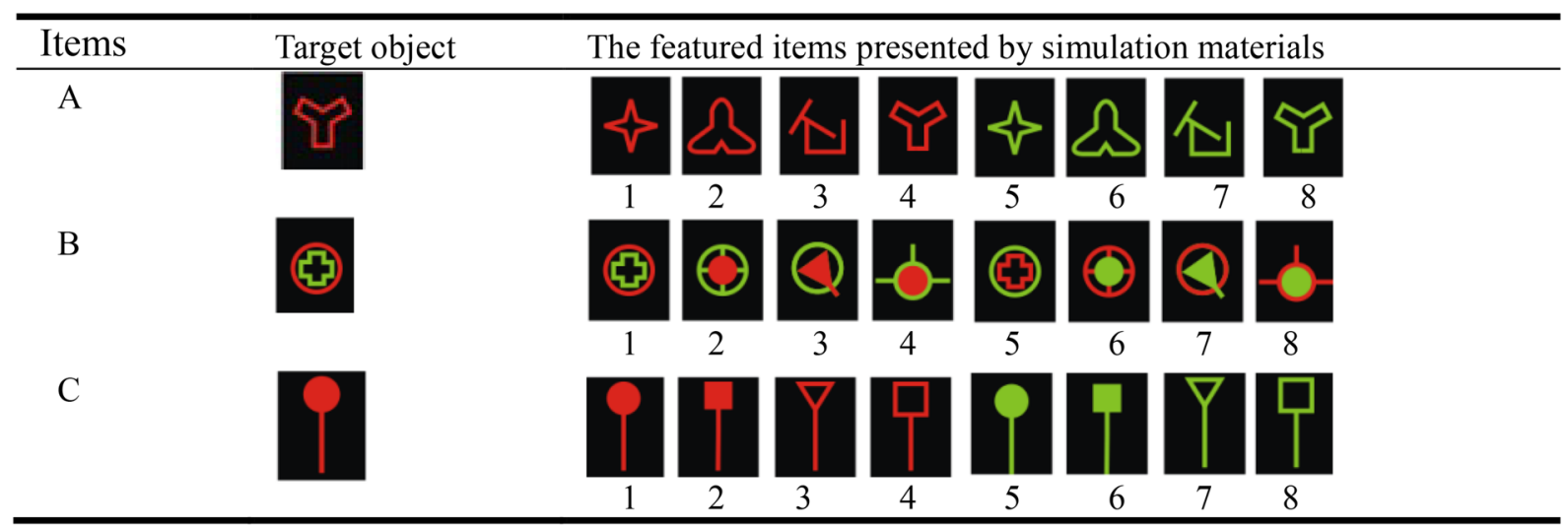

the gaze on the target object when distinguishing two featured items of B-1 and B-4. It explained that during the target search the subject showed a slightly longer fixation time on similar target objects and the similarity of shapes that had influenced the recognition of target objects.

\section{Application}

According to the results of experiment, the radar situation interface could be optimized from the points of information feature, the information capacity and the position of information blocks. Figure 12 has shown an optimized situation interface display which have been adjusted some information blocks to decrease interference factor, and the feature items should be displayed to users with regular-shape single color. This optimized project could offer a rational reference for the rational layout and improvement of the radar situation-interface of fighter. The conclusions also can be intended for the improved design of information matter features.

\section{Conclusions}

(1) Interference environment and the feature of information matter are two major factors in radar situationinterface that influence the information matter identification, which should be considered in information layout of complex situation-interface as a design factor.

(2) Information identification under low and high interference environments shows significant difference. Various information presented in radar situationinterface has formed a high-interference environment. Pilots could be able to precisely identify the information only in a forcibly interference-free environment.

(3) In radar situation-interface different information matters constitute three kinds of different feature items, their reaction time, and error rate, which pre-

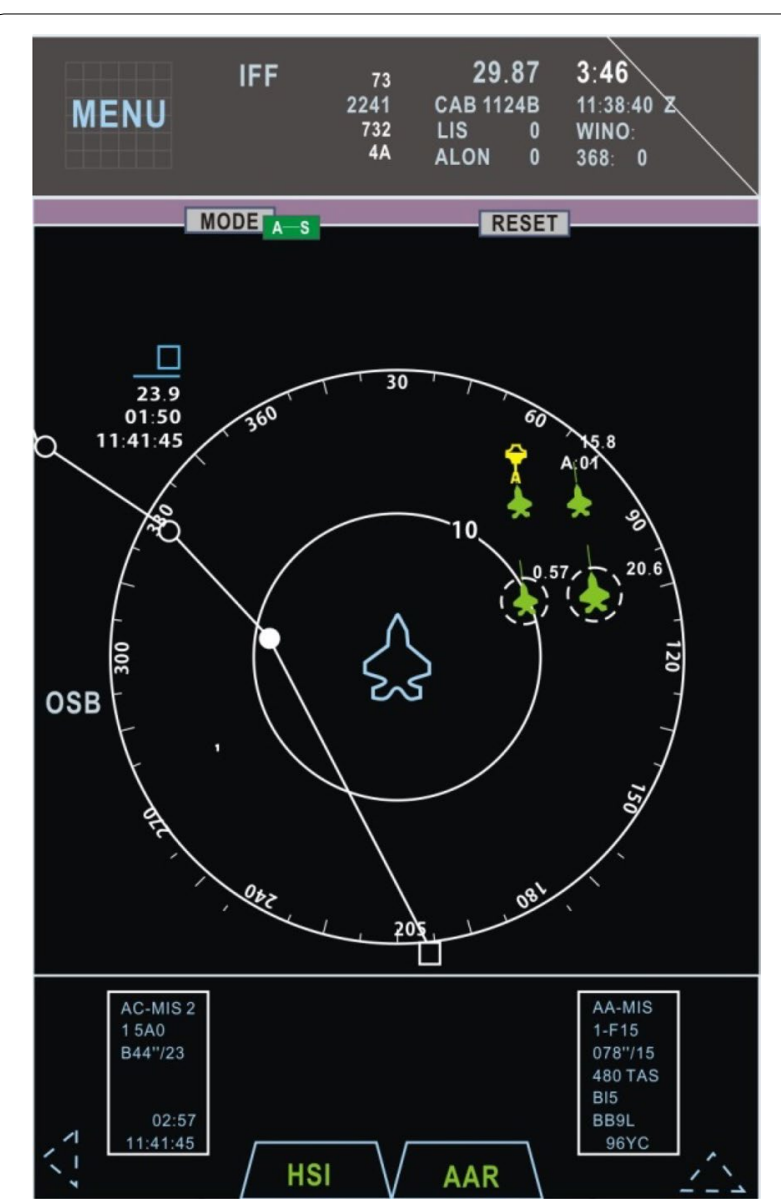

Figure 12 An optimized situation interface display

sent obvious regular changes. The reaction time of three feature items (regular-shape single color, irregular-shape single color and irregular-shape hybrid color) reveals a trend of progressive increase, suggests that an irregular-shaped hybrid color feature 
item are characterized by identification difficulty and long reaction time.

(4) The eye movement data also suggested that the target search under high interference environment displayed the longer fixation time, more saccade counts and longer scan path, than under low interference environment. In addition, the first saccade time, the total fixation time and saccade counts of featured items search presented an increasing trend, which is the same as reaction.

\section{Authors' Contributions}

X-LW was in charge of the whole trial; X-LW wrote the manuscript; $J$ and FZ assisted with sampling and laboratory analyses. All authors have read and approved the final manuscript.

\section{Author details}

${ }^{1}$ China College of Mechanical and Electrical Engineering, Hohai University, Changzhou 213022, China. ${ }^{2}$ College of Furnishings and Industrial Design, Nanjing Forestry University, Nanjing 211189, China.

\section{Authors' Information}

Xiao-li Wu, born in 1980, PhD, is currently an associate professor and a Master supervisor, major research in Human-computer Interaction. She currently is working in College of Mechanical and Electrical Engineering, Hohai University, China. And she graduated from School of Mechanical Engineering, Southeast University, China. Her research is focused on Human-computer Interaction and design-cognition.

Jing Li, born in 1987, PhD, a lecturer at College of Furnishings and Industrial Design, Nanjing Forestry University, China. And she graduated from School of Mechanical Engineering, Southeast University, China.

Feng Zhou, is currently an associate professor at College of Mechanical and Electrical Engineering, Hohai University, China. Graduated from School of Knowledge Science, Advanced Institute of Science and Technology, Japan. Research interests include design computing and cognition.

\section{Competing Interests}

The authors declare no competing financial interests.

\section{Funding}

Supported by National Natural Science Foundation of China (Grant Nos. 71601068, 61603123), Science and Technology of Changzhou, China (Grant No. CE20175032), Jiangsu Provincial Key Project of Philosophy and the Social Sciences of China (Grant No. 2017ZDIXM023), Outstanding Young Scholars Joint Funds of Chinese Ergonomics-King Far (Grant No. 2017-5), Overseas Research Project of Jiangsu Province (Grant No. 2017), and Fundamental Research Funds for the Central Universities of China (Grant Nos. 2015B22714).

\section{Publisher's Note}

Springer Nature remains neutral with regard to jurisdictional claims in published maps and institutional affiliations.

Received: 23 September 2016 Accepted: 30 May 2018 Published online: 08 June 2018

\section{References}

[1] MarkT M. Usable advanced visual interfaces in aviation. Proceedings of the International Working Conference on Advanced Visual Interfaces, ACM. New York, USA, 2012: 2-3.

[2] Heather F Neyedli, Justin G Hollands, Greg A Jamieson. Beyond identity: Incorporating system reliability information into an automated combat identification system. Human Factors: The Journal of the Human Factors and Ergonomics Society, 2011, 53(4): 338-355.
[3] D M Zhuang, Y X Yan. Encoding of aircraft cockpit display interface. Journal of Nanjing University of Aeronautics \& Astronautics, 2009, 41 (4): 466-469. (in Chinese)

[4] L Zhang, D M Zhuang, Color matching of aircraft interface design. Journal of Beijing University of Aeronautics \& Astronautics, 2009, 35(8): 1001-1004. (in Chinese)

[5] L Zhang, D M Zhuang, Text and position coding of human-machine display interface. Journal of Beijing University of Aeronautics \& Astronautics, 2011, 37(2): 185-188. (in Chinese)

[6] L Zhang, D M Zhuang, X Wanyan. Information coding for cockpit humanmachine interface. Chinese Journal of Mechanical Engineering, 2011, 24(4): 707-712.

[7] L Tong, P Yan, F Liu. Monitoring computer numerical control machining progress based on information fusion. Chinese Journal of Mechanical Engineering, 2011, 24(6): 1074-1082.

[8] HYWang, T Bian, C Q Xue. Experimental evaluation of fighter's interface layout based on eye tracking. Electro-Mechanical Engineering, 2011, 27(6): 50-53. (in Chinese)

[9] HYWang, T Bian, C Q Xue. Layout design of display interface for a new generation fighter. Electro-Mechanical Engineering, 2011, 27(4): 57-61. (in Chinese)

[10] Q Liu. Research on design and experiment method of avionics system display interface. Nanjing: School of Mechanical Engineering, Southeast University, 2012. (in Chinese)

[11] J Li, C Q Xue, HY Wang, et al. Information encoding in human-computer interface on the equilibrium of time pressure. Journal of Computer Aided Design \& Computer Graphics, 2013, 25(7): 1022-1028. (in Chinese)

[12] J Li, C Q Xue, M H Shi, et al. Information visual structure based on multidimensional attributes of information. Journal of southeast University (Natural Science Edition), 2012, 42(6): 1094-1099. (in Chinese)

[13] L Zhou, C Q Xue, Tomimatsu, et al. Research of interface composition design optimization based on visual balance. The 2013 International Conference on Intelligent Systems and Knowledge Engineering, Shenzhen, 2013, November 20-23, 279: 483-493.

[14] L Zhou, C Q Xue, W C Tang, et al. Aesthetic evaluation method of interface elements layout design. Journal of Computer-Aided Design \& Computer Graphics, 2013, 25(5): 758-766. (in Chinese)

[15] T Jin, C Q Xue, et al. A new method of building an evaluation model for User Interface. 3rd International Conference on Machinery, Materials Science and Engineering Applications, Wuhan, China, 2013, June 20-12, 2013, 744: 605-609.

[16] T Jin, C Q Xue, HY Wang, et al. Research on evaluating methods of situation awareness of digital graphical interfaces. Chinese Journal of Engineering Design, 2014, 21(1): 87-91. (in Chinese)

[17] X L Wu. Error-cognition mechanism of task interface in complex information system. Beijing: Science Press, 2017. (in Chinese)

[18] X L Wu. Research on the mechanism of error-cognition for interaction interface of complex information. Nanjing: Southeast University, 2015. (in Chinese)

[19] X L Wu, C Q Xue, F Zhou. Misperception model-based analytic method of visual interface design factors. Engineering Psychology and Cognitive Ergonomics - 11th International Conference, EPCE 2014, Held as Part of HCI International 2014, Proceedings, Crete, Greece, June 22-27: 8532 LNAI, 284-292.

[20] X L Wu, C Q Xue, H Y Wang. E-C mapping model of human-computer interaction interfaces of complicated systems. Journal of Mechanical Engineering, 2014, 50(12): 206-212. (in Chinese)

[21] X L Wu, C Q Xue, W C Tang, et al. Experimental study on visual limitation experiment of goal-seeking in radar situation-interface. Journal of Southeast University (Natural Science Edition), 2014, 44(6): 1166-1170. (in Chinese)

[22] X L Wu, Y Chen, F Zhou. An interface analysis method of complex information system by introducing error factors. 13th International Conference on Engineering Psychology and Cognitive Ergonomics, EPCE 2016 and Held as Part of 18th International Conference on Human-Computer Interaction, HCl International, Toronto, Canada, July 17-22, 9736 LNAI: 116-124.

[23] X L Wu, C Q Xue, W C Tang. Study on eye movement of information omission misjudgment in radar situation-interface. Engineering Psychology and Cognitive Ergonomics - 11th International Conference, EPCE 2014, Held as Part of HCl International 2014, Proceedings, Crete, Greece, June 22-27, 8532 LNAI: 407-418. 
[24] Y F Niu, C Q Xue. Icon memory research under different time pressures and icon quantities based on event-related potential. Journal of Southeast University (English Edition), 2014, 30(1): 45-50.

[25] C Q Xue, X L Wu, Y F Niu, et al. Brain mechanism research on visual information cognition of digital human computer interface. Communications in Computer an Information Science-HCl International 2015-Posters' extended abstracts, PT1, Los Angeles, CA, USA, August 2-7, 2015, 528 : $144-149$

[26] C Q Xue, J Li, H Y Wang, et al. Effects of target and distractor saturations on the cognitive performance of an integrated display interface. Chinese Journal of Mechanical Engineering, 2015, 28(1): 208-216.

[27] J Theeuwes, R Burger. Attentional control during visual search the effect of irrelevant singletons. Journal of Experimental Psychology: Human Perception and Performance, 1998, 24: 1342-1353.

[28] J Theeuwes. Top-down search strategies cannot override attentional capture. Psychonomic Bulletin \& Review, 2004, 11(1): 65-70.

[29] M D Fleetwood, M D Byrne. Modeling icon search in ACT-R/PM. Cognitive Systems Research, 2002, 3: 25-33.

[30] M D Fleetwood, M D Byrne. Modeling the visual search of displays: A revised ACT-R/PM model of icon search based on eye-tracking and experimental data. Human-Computer Interaction, 2006, 21(2): 153-197.
[31] Patrick Monnier. Redundant coding assessed in a visual search task. Displays, 2003, 24(1): 49-55

[32] B L Yu. An experimental study of effects of character spacing under visual interference. Psychological Science, 1994, 17(3): 129-132. (in Chinese)

[33] Y Luo. Exploration to manner of coding of visual object information. Chong Qing: Development and Education Psychology of Southwest University, 2010. (in Chinese)

[34] K FVan Orden, J Divita, M J Shim. Redundant use of luminance and flashing with shape and color as highlighting codes in symbolic displays. Human Factors, 1993, 35: 195-204.

[35] C D Wickens, A D Andre. Proximity compatibility and information display: effects of color, space, and object display on information integration. Human Factors, 1990, 32(1): 61-77.

[36] Y Michelle, C D Wickens. Attentional filtering in the design of electronic map displays: A comparison of color coding, intensity coding, and decluttering techniques. Human Factors, 2001, 43(4): 543-562.

\section{Submit your manuscript to a SpringerOpen ${ }^{\circ}$ journal and benefit from:}

- Convenient online submission

- Rigorous peer review

- Open access: articles freely available online

- High visibility within the field

- Retaining the copyright to your article

Submit your next manuscript at springeropen.com 